\title{
Complete response after radiation-assisted immunotherapy for metastatic urothelial carcinoma: a case report and literature review
}

\author{
Cheng-Sheng Liu ${ }^{1}$, Chien-Jui Cheng ${ }^{2,3}$, Yun-Ru Liu ${ }^{4}$, Shauh-Der Yeh ${ }^{5}$, Shiu-Chen Jeng ${ }^{1}$, \\ Long-Sheng $\mathrm{Lu}^{1,6,7,8}$, Jeng-Fong Chiou, ${ }^{1,9,10}$
}

${ }^{1}$ Department of Radiation Oncology, Taipei Medical University Hospital, Taipei, Taiwan; ${ }^{2}$ Department of Pathology, School of Medicine, College of Medicine, Taipei Medical University, Taipei, Taiwan; ${ }^{3}$ Department of Pathology, Taipei Medical University Hospital, Taipei, Taiwan; ${ }^{4}$ Joint Biobank, Office of Human Research, Taipei Medical University, Taipei, Taiwan; ${ }^{5}$ Department of Urology, ${ }^{6}$ Translational Laboratory, Department of Medical Research, Taipei Medical University Hospital, Taipei, Taiwan; ${ }^{7}$ Graduate Institute of Biomedical Materials and Tissue Engineering, College of Biomedical Engineering, Taipei Medical University, Taipei, Taiwan; ${ }^{8}$ International Ph.D. Program in Biomedical Engineering, College of Biomedical Engineering, Taipei Medical University, Taipei, Taiwan; ${ }^{9}$ Taipei Cancer Center, Taipei Medical University, Taipei, Taiwan; ${ }^{10}$ Department of Radiology, School of Medicine, College of Medicine, Taipei Medical University, Taipei, Taiwan

Correspondence to: Long-Sheng Lu, MD, PhD. No. 252, Wuxing St., Xinyi Dist., Taiepi City 110, Taiwan. Email: 1slu@tmu.edu.tw.

\begin{abstract}
Immunotherapy is emerging to be a new effective option in patients with metastatic urothelial carcinoma (UC) who are cisplatin-ineligible. However the feasibility of combinatory strategy in this setting is unknown. We reported our experience of treating one case of metastatic UC with radiation-assisted immunotherapy (RAIT). A 69-year-old male patient with end stage renal disease is a case of metastatic UC. The tumor was microsatellite stable. He was treated with six doses of triweekly pembrolizumab in four months. Hypofractionated radiotherapy to all metastatic lymphadenopathies (LAPs) was delivered between the second and the third dose of pembrolizumab. Complete response was confirmed with positron emission tomography/computed tomography one month after treatment. Metachronous bladder cancer was noticed nine months later and was successfully treated with transurethral resection of bladder tumor followed by adjuvant Bacillus Calmette-Guerin intravesical instillation. Genetic profiling from next generation sequencing (NGS) revealed high mutational burden (65.2 mutations/megabase) and CDH1 mutation. He remains disease free and dialysis free one and half year after treatment. Furthermore, circulating tumor cells (CTCs) dynamics correlates with the disease course precisely. This is a case of exceptional response and treating metastatic UC with RAIT in cisplatin-ineligible patients may warrant further investigation.
\end{abstract}

Keywords: Hypofractionated radiotherapy; metastatic urothelial carcinoma; radiation-assisted immunotherapy (RAIT)

Received: 14 March 2020; Accepted: 19 August 2020; Published: 30 September 2020.

doi: $10.21037 /$ tro-20-30

View this article at: http://dx.doi.org/10.21037/tro-20-30

\section{Introduction}

Cisplatin-based chemotherapy is the standard treatment for metastatic urothelial carcinoma (UC) with the progressionfree survival (PFS) of 7.4 months and the overall survival (OS) of 14.0 months (1). However, approximately half of these patients are cisplatin-ineligible due to renal function impairment, poor performance status or other comorbidities.
Immune checkpoint blockades that target the programmed death-1 (PD-1)/programmed death-Ligand 1 (PD-L1) pathway have shown an inspiring result and were approved by Food and Drug Administration (FDA) for patients with locally advanced and metastatic UC. Radiotherapy to tumor cells can stimulate $\mathrm{CD} 8^{+} \mathrm{T}$ lymphocytes in tumor microenvironment and this may result in tumor response. 


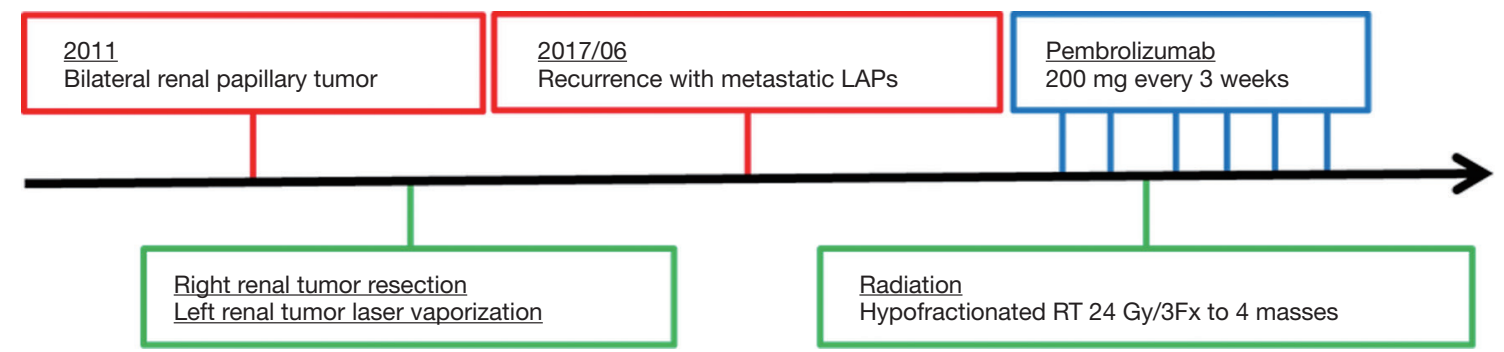

Figure 1 Treatment course of radiation-assisted immunotherapy. Pembrolizumab (200 mg) was administrated every 3 weeks for 6 courses and hypofractionated radiotherapy to lymphadenopathies was delivered between the second and third dose of immunotherapy.

It has been reported that hypofractionated radiotherapy to oligometastatic tumor was associated with significantly improved 5-year OS from $28 \%$ to $41 \%$ (2). Therefore, it is a reasonable approach to combine radiotherapy with immunotherapy (radiation-assisted immunotherapy, RAIT) for cisplatin-ineligible patients with oligometastatic UC. Here, we reported our experience of treating one such case with RAIT. We present the following case in accordance with the CARE reporting checklist (available at http:// dx.doi.org/10.21037/tro-20-30).

\section{Case presentation}

A male patient with past medical history of chronic kidney disease, stage 5 , and type 2 diabetes mellitus was diagnosed as bilateral ureteral papillary tumor in 2011 at 63 years of age. The patient refused bilateral nephroureterectomy and received right renal tumor resection and left renal tumor laser vaporization instead. There was no residual nor recurrent tumor at his upper urinary tract in the following studies.

He suffered from low back and left flank pain in June, 2017 and abdominal magnetic resonance imaging (MRI) showed lymphadenopathies (LAPs) up to $4 \mathrm{~cm}$ in left retrocrural \& para-aortic regions. The para-aortic lymph node taken from computed tomography-guided percutaneous biopsy confirmed the diagnosis of metastatic carcinoma. Further immunohistochemistry studies revealed these tumor cells were positive for CK7, GATA3 and 34BE12 and negative for CK20. These results were consistent with a diagnosis of metastatic UC to para-aortic lymph node. Positron emission tomography/computed tomography (PET/CT) showed lymph node metastases in the left supra-clavicular fossa, left mediastinum and paraaortic areas without urinary tract lesions. His hemogram and serum biochemistry were anemic (hemoglobin: $9.8 \mathrm{~g} / \mathrm{dL}$ ) with elevated creatinine level (creatinine: $8.0 \mathrm{mg} / \mathrm{dL}$ ). Molecular pathology analysis revealed one of microsatellite markers (MONO-27) is altered, and the tumor was classified as microsatellite instability-low (MSI-L) but the sample was insufficient for further next generation sequencing (NGS). Count of $\mathrm{EPCAM}^{+} \mathrm{CK}^{+} \mathrm{CD} 45^{-}$nucleated cells (circulating tumor cells, CTCs) was enumerated with IsoFlux system (Fluxion, CA) and there were 40 CTCs per $7.5 \mathrm{~mL}$ venous blood sample before treatment.

The patient received pembrolizumab at a dose of $200 \mathrm{mg}$ every 3 weeks, for six courses in four months (Figure 1). Hypofractionated radiotherapy (24 Gray in 3 fractions, via $6 \mathrm{MV}$ photons) to all metastatic LAPs was delivered between the second and third dose of pembrolizumab as cancerdirected treatment (Figure 2). All procedures performed in studies involving human participants were in accordance with the ethical standards of the institutional and/or national research committee(s) and with the Helsinki Declaration (as revised in 2013). Written informed consent was obtained from the patient. At the end of treatment, a PET/CT scan showed complete response (Figure 3). The CTCs count decreased to 16 per $7.5 \mathrm{~mL}$ venous blood sample (Figure 4). During the treatment, grade 1 side effects including fatigue and pruritus were noticed. Serum creatinine rose from 8.4 to $9.2 \mathrm{mg} / \mathrm{dL}$ without uremic symptoms (Figure 4).

The disease remained in complete response nine months after treatment when CTC count got increased to 22 per $7.5 \mathrm{~mL}$ venous blood sample. A new polypoid lesion at right posterior bladder wall was found during pelvic MRI two months later without upper urinary tract involvement. Transurethral resection of bladder tumor (TUR-BT) revealed a non-muscle invasive high grade bladder cancer. NGS finding suggested high mutation burden (65.2 mutations/MB) and incidentally found CDH1 mutation (Figure 5). He was treated with immediately intravesical mitomycin $\mathrm{C}$ and adjuvant intravesical Bacillus Calmette-Guerin (BCG). He 

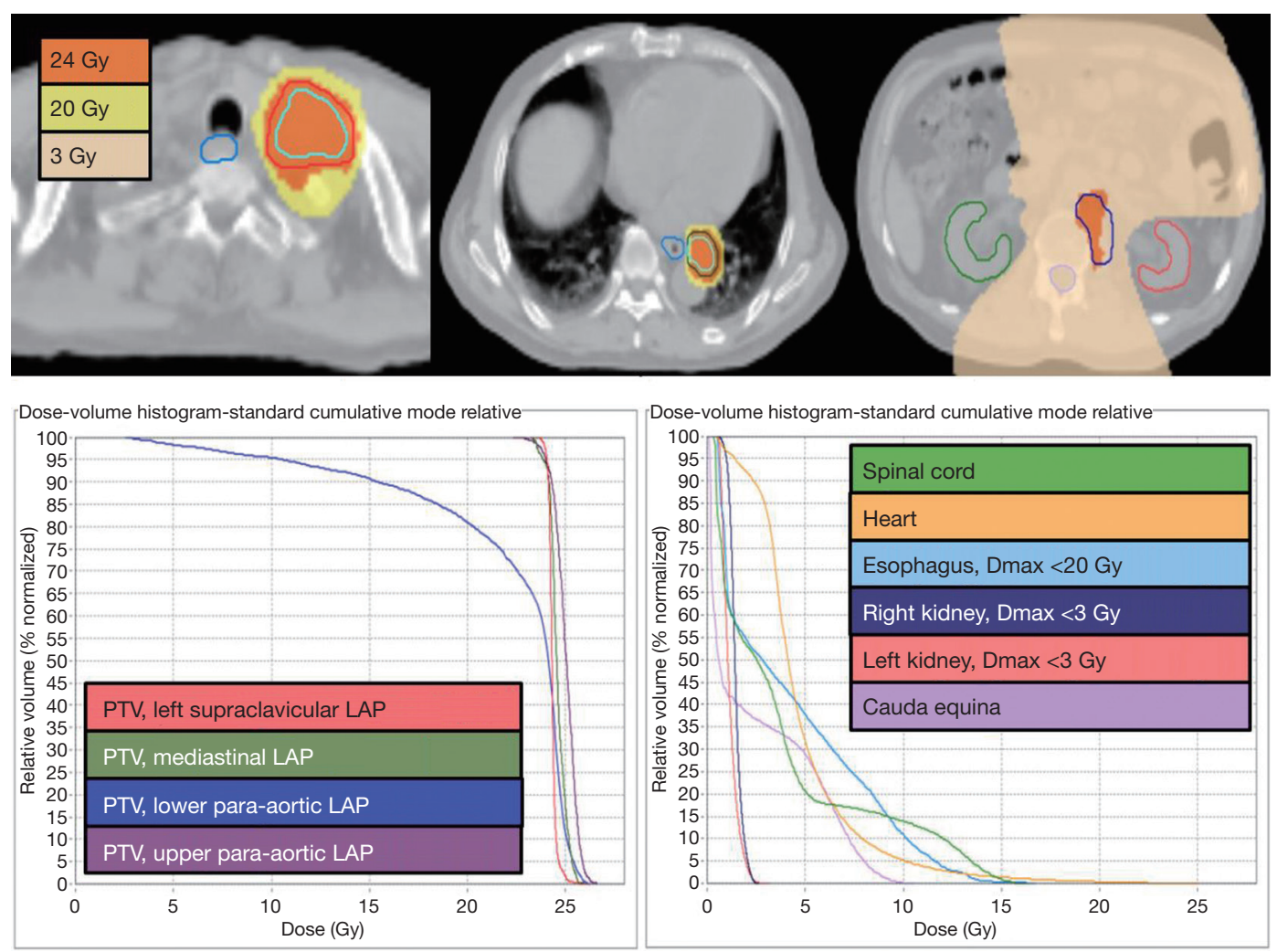

Figure 2 Irradiation was delivered to all metastatic lymphadenopathies (LAPs) over left supraclavicular area, left mediastinum, and paraaortic area. Radiation contour, dose distribution, dose volume histogram of treatment site and organs at risk. The sky blue line represented clinical target volume. The red, green, and blue lines represented plan target volume (PTV) of supraclavicular, mediastinal, para-aortic LAPs respectively. The volume receiving $97 \%$ or more prescribed dose, V97, of left supraclavicular, mediastinal, upper para-aortic LAP were more than $99 \%$. The V97 of lower para-aortic LAP was $71.1 \%$ due to its proximity to left kidney. The organ at risk including esophagus and bilateral kidney in this plain received less than 20 Gy, and less than 3 Gy respectively.

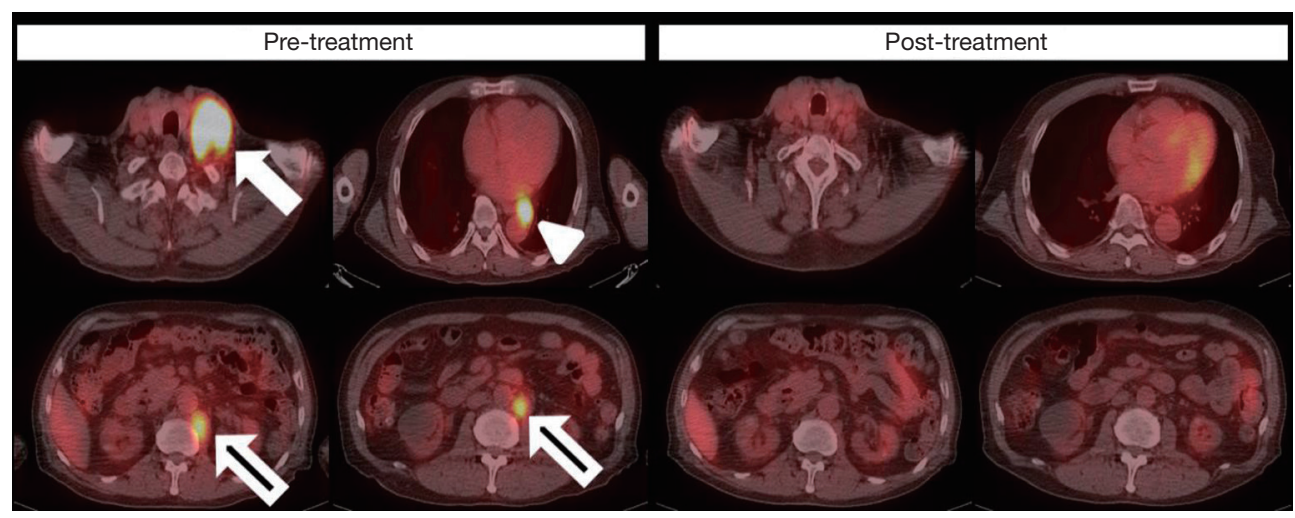

Figure 3 Pre-treatment and post-treatment PET/CT scan. Pre-treatment scan revealed metastatic lymphadenopathies in the left supraclavicular fossa (close arrow), and para-aortic areas in the left mediastinum (arrowhead) and abdomen (open arrow). One month posttreatment scan revealed complete response and no evidence of residual diseases in the whole body scan. 

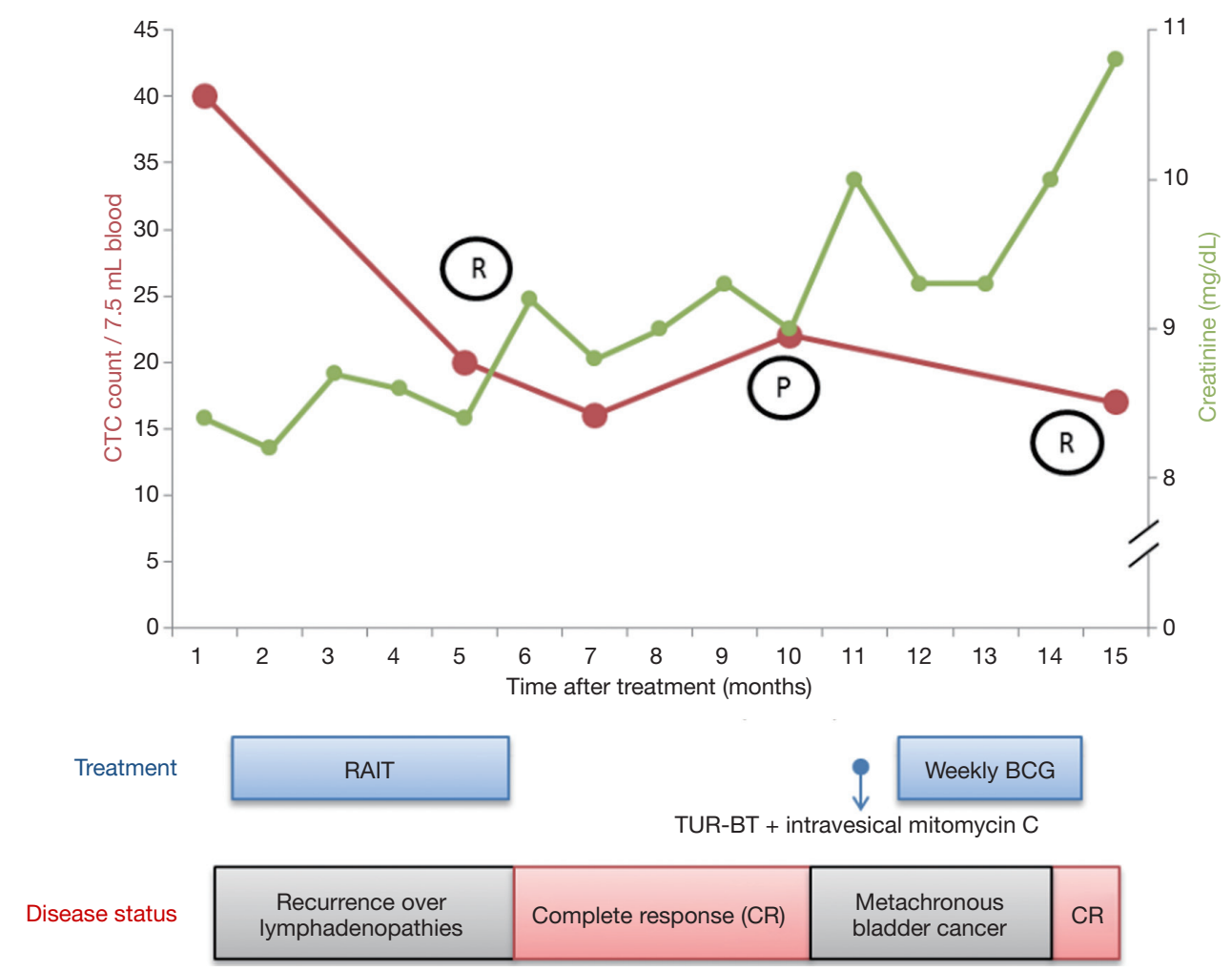

Figure 4 Serial monitoring CTCs, changes of serum creatinine, treatment history and clinical responses are noted in the plot. The red line indicated CTC counts in 7.5 venous blood sample which correlated with disease course. The green line indicated creatinine level (mg/dL) and there was Grade 1 creatinine elevation. P, disease progression; R, treatment response; CTCs, circulating tumor cells.

started peritoneal dialysis 17 months after RAIT.

\section{Discussion}

Cisplatin-based chemotherapy is the first-line treatment option for advanced UC. For patients who are cisplatinineligible, carboplatin-based chemotherapy, gemcitabine, and paclitaxel were alternative choices. However, toxicities and limited efficacy are still a major concern with these approaches. Erdafitinib, a tyrosine kinase inhibitor of fibroblast growth factor receptor (FGFR) 1-4, was recently approved by FDA and shows potent anti-tumor activity for previously treated locally advanced or metastatic UC with the response rate of $40 \%$ (3). A phase III investigation is now ongoing comparing the efficacy of erdafitinib, vinflunine or docetaxel or pembrolizumab in previously treated locally advanced and metastatic UC patients with FGFR gene aberrations.

PD-1 and cytotoxic T-lymphocyte-associated protein 4 (CTLA-4) are both immune-modulatory molecules which act as immune checkpoint. The phase II study KEYNOTE 052 demonstrated that first-line pembrolizumab prolongs OS from 7.4 to 10.3 months for patients with metastatic UC who are cisplatin-ineligible (4). Atezolizumab, an anti-PD-L1 antagonist, also shows durable response in untreated, cisplatin-ineligible, metastatic UC (5). In addition, PD-L1 status and tumor mutation load are associated with higher frequency of treatment response. Both of the immune checkpoint blockade are well tolerated with the renal toxicity is mild as three of the 489 patients were reported to have treatment-related renal failure. Further clinical trial KEYNOTE 361 was designed to determine the efficacy and safety of pembrolizumab with or without chemotherapy as compared with chemotherapy alone in metastatic UC.

Tumor microenvironment plays an important role in treating cancers with immunotherapy. The interactions between $\mathrm{T}$ cells, dendritic cells, tumor cells regulate the activation of T-cells. Several studies demonstrated different biomarkers may predict the responses of immune 


\begin{tabular}{|c|c|c|c|c|c|c|c|}
\hline & \multicolumn{6}{|c|}{ Variant(s) with clinical relevance } & \\
\hline & \multicolumn{6}{|c|}{ Single nucleotide and small indel variants } & \\
\hline & \multicolumn{2}{|c|}{\begin{tabular}{l|l} 
Gene & \\
\end{tabular}} & Amino acid change & \multicolumn{2}{|c|}{ Coverage } & Allele frequency & \\
\hline & \multicolumn{2}{|l|}{ CDKN2C } & R68 & \multicolumn{2}{|c|}{1042} & $40.3 \%$ & \\
\hline & \multicolumn{2}{|l|}{ CHEK2 } & K312 & \multicolumn{2}{|c|}{598} & $39.5 \%$ & \\
\hline & \multicolumn{2}{|l|}{ HRAS } & Q61L & \multicolumn{2}{|c|}{620} & $38.5 \%$ & \\
\hline & \multicolumn{2}{|l|}{ PTPRD } & Splice donor & \multicolumn{2}{|c|}{430} & $39.8 \%$ & \\
\hline & \multicolumn{6}{|c|}{ Tumor mutational burden } & \\
\hline & \multicolumn{6}{|c|}{65.2 mutations/megabase (high) } & \\
\hline & \multicolumn{6}{|c|}{ Microsatellite instability } & \\
\hline & \multicolumn{6}{|l|}{ MSS } & \\
\hline \multicolumn{4}{|c|}{ Variant(s) of unknown significance } & MUC16 & W536R & 2230 & $14.8 \%$ \\
\hline Single nucle & de and small indel & ints gene & & MUC16 & L1684H & 1965 & $13.1 \%$ \\
\hline Gono & Amino acid & Coverage & Allele & MUC6 & S2201T & 1492 & $36.1 \%$ \\
\hline Gene & change & Coverage & frequency & NBN & I558L & 998 & $61.8 \%$ \\
\hline ADAMTS13 & I110F & 1247 & $37.4 \%$ & NFE2L2 & P128S & 470 & $49.4 \%$ \\
\hline ADAMTS16 & Splice region & 1312 & $37.5 \%$ & NOTCH1 & Y358F & 309 & $36.9 \%$ \\
\hline ARID1A & Q1552L & 604 & $40.7 \%$ & NTRK1 & V99M & 799 & $50.9 \%$ \\
\hline ARID1B & Splice region & 734 & $38.6 \%$ & NTRK1 & D306E & 645 & $34.1 \%$ \\
\hline$A S X L 1$ & K586 & 582 & $33.0 \%$ & NTRK2 & YG400 & 947 & $41.2 \%$ \\
\hline ATM & $\mathrm{N} 485 \mathrm{Y}$ & 628 & $35.8 \%$ & NTRK2 & Splice acceptor & 618 & $40.8 \%$ \\
\hline$B L M$ & Q1210L & 445 & $39.8 \%$ & NTRK3 & Q255L & 927 & $38.5 \%$ \\
\hline CD19 & M551L & 684 & $34.6 \%$ & NTRK3 & K346M & 932 & $35.9 \%$ \\
\hline $\mathrm{CDH1}$ & E864K & 819 & $50.5 \%$ & PAX5 & Y388N & 889 & $38.8 \%$ \\
\hline CDKN1B & K134M & 874 & $35.8 \%$ & PDGFRA & S783T & 714 & $42.0 \%$ \\
\hline CHEK2 & K141E & 307 & $35.5 \%$ & PIK3C2G & Y634 & 402 & $44.3 \%$ \\
\hline CREBBP & Splice acceptor & 867 & $34.5 \%$ & POLB & K230 & 1039 & $37.4 \%$ \\
\hline CYP2B6 & P167A & 119 & $49.6 \%$ & PRKDC & R2529W & 825 & $24.7 \%$ \\
\hline$D D R 2$ & S449C & 1164 & $38.0 \%$ & PTPRD & Y1897N & 552 & $41.3 \%$ \\
\hline DTX1 & D394E & 613 & $37.2 \%$ & PTPRD & E118V & 616 & $40.3 \%$ \\
\hline E2F3 & Q144L & 892 & $40.0 \%$ & PTPRD & G1478R & 981 & $38.6 \%$ \\
\hline E2F3 & K133M & 885 & $34.4 \%$ & RAD54L & $\mathrm{Y} 121 \mathrm{C}$ & 382 & $38.0 \%$ \\
\hline EGFR & $\mathrm{R} 252 \mathrm{C}$ & 1740 & $43.4 \%$ & $R E T$ & K666 & 943 & $41.0 \%$ \\
\hline EPHA7 & E56V & 709 & $35.3 \%$ & ROS1 & Y1021S & 419 & $39.1 \%$ \\
\hline$E Z H 2$ & R33 & 202 & $38.6 \%$ & RUNX1T1 & Splice region & 979 & $22.0 \%$ \\
\hline FANCD2 & Q552L & 1174 & $33.6 \%$ & SMARCB1 & Q244L & 752 & $41.2 \%$ \\
\hline FOXL2 & S238C & 238 & $50.4 \%$ & SMARCB1 & Y313F & 669 & $38.4 \%$ \\
\hline GRIN2A & Splice acceptor & 854 & $37.0 \%$ & SYNE1 & V3890I & 700 & $51.7 \%$ \\
\hline HIST1H1C & A24V & 381 & $48.8 \%$ & SYNE1 & T5957P & 720 & $35.0 \%$ \\
\hline HNF1A & Q350H & 690 & $38.3 \%$ & TAP2 & G47R & 1090 & $48.2 \%$ \\
\hline INPP4B & Q857L & 1031 & $36.4 \%$ & TEK & Y1024F & 1332 & $49.8 \%$ \\
\hline KDM5A & $\mathrm{K} 55 \mathrm{M}$ & 1384 & $35.4 \%$ & TERT & Q770L & 390 & $40.5 \%$ \\
\hline KEAP1 & Q62L & 683 & $73.8 \%$ & TET1 & Splice region & 778 & $34.7 \%$ \\
\hline KIT & Y259N & 1045 & $38.7 \%$ & TGFBR2 & Q306L & 1451 & $53.5 \%$ \\
\hline KMT2D & E3078V & 278 & $36.3 \%$ & TSHR & Y521F & 767 & $41.1 \%$ \\
\hline LRP1B & E2499V & 481 & $42.2 \%$ & USH2A & R2001C & 607 & $50.9 \%$ \\
\hline MET & Y1177F & 802 & $42.3 \%$ & USH2A & Y2179C & 603 & $40.6 \%$ \\
\hline MTOR & R2086W & 956 & $36.9 \%$ & USH2A & Y3807N & 1030 & $37.7 \%$ \\
\hline MUC16 & L5485Q & 2115 & $78.9 \%$ & USH2A & F2353S & 851 & $35.5 \%$ \\
\hline
\end{tabular}

Figure 5 Genetic profiles of the tumor samples from transurethral resection of bladder tumor. High mutation burden indicates the possibility of beneficial from immune checkpoint blockade. CDH1 variants with allele frequency around $50 \%$ may be related to the secondary gastric cancer diagnosed two years after the diagnosis of metastatic urothelial carcinoma. 
Table 1 Reported four cases of upper urogenital tract cancer treated with radiation-assisted immunotherapy (RAIT)

\begin{tabular}{|c|c|c|c|c|c|c|c|}
\hline Case & References & $\begin{array}{l}\text { Age }(y) \\
\text { sex }\end{array}$ & $\begin{array}{l}\text { Cancer } \\
\text { Histology }\end{array}$ & $\begin{array}{l}\text { Radiotherapy } \\
\text { (RT) }\end{array}$ & $\begin{array}{l}\text { Immunotherapy } \\
\text { (IO) }\end{array}$ & $\begin{array}{c}\text { Time sequence of } \\
\text { IO \& RT }\end{array}$ & Response \\
\hline 1 & Xie et al. (14) & $54, \mathrm{M}$ & $\mathrm{RCC}$, clear cell & $32 \mathrm{~Gy} / 4 \mathrm{Fx}$ & Pembrolizumab & Before \& after & $\mathrm{CR}$ \\
\hline 2 & van Gysen et al. (15) & $66, F$ & RCC, clear cell & 36 Gy/12 Fx & Nivolumab & Before & PR \\
\hline 3 & LaPlant et al. (16) & $24, \mathrm{M}$ & $\mathrm{RCC}$, clear cell & 27 Gy/3 Fx & $\begin{array}{l}\text { Nivolumab \& } \\
\text { Ipilimumab }\end{array}$ & Before \& after & PR \\
\hline
\end{tabular}

$\mathrm{RCC}$, renal cell carcinoma; CR, complete response; PR, partial response; PD, progressive disease.

checkpoint blockade. PD-L1 expression on tumor cells had been studied widely as a biomarker of response to immunotherapy. However, conflicting results were found between different studies $(6,7)$. The other pathology information from tumor samples such as tumor infiltrative lymphocytes including cytotoxic and memory $\mathrm{T}$ cells, microsatellite instability, tumor mutation burden are associated with clinical outcomes (8-11). In addition, some of the serum biomarkers are associated with favorable treatment response of immunotherapy. These biomarkers include low lactate dehydrogenase level and high absolute or relative lymphocyte counts. However, there was no consensus about the cut-off value of each biomarker to predict clinical outcome. Furthermore, none of the above serum or pathology information could be used solely to predict treatment response after receiving immune checkpoint blockade. Hence, it is essential to establish robust biomarkers for treating cancer patients toward precision immune-oncology.

Traditionally, the role of radiotherapy for metastatic UC was to provide symptomatic palliation. However in the era of immuno-oncology, radiotherapy as an immune adjuvant may be considered to be an alternative curative approach. Irradiation to tumor cells can lead to the release of damageassociated molecular pattern (DAMP) molecules, such as high mobility group protein box 1 (HMGB1), adenosine triphosphate (ATP) and calreticulin (CRT). Also tumor irradiation will lead to intratumoral recruitment of tumorreactive $T$ cells as well as matured dendritic cells. The phenomenon is especially pronounced with a fraction size larger than 7.5 Gray. Together, these effects lead to insitu vaccination and promote tumor control (12). RAIT is now under investigation towards a wide range of tumors in several early phase trials. In this report, we describe a case of exceptional response to RAIT in metastatic UC. While RAIT with a similar protocol has been reported in a phase I study for treating 18 cases of metastatic bladder cancers, for upper urogenital tract cancers there were only 4 cases with renal cell carcinoma reports so far with satisfactory safety profiles (13-17) (Table 1). It is worth investigation if success experience of RAIT for bladder cancers and be extrapolated to metastatic upper genitourinary tract cancers that are cisplatin-ineligible.

In the era of precision medicine, genetic profiling from NGS sometimes provides possible treatment options and surveillance strategy. However, only FGFR genetic alteration was suggested while treating patients with locally advanced or metastatic UC. In our case, the pathology sample retrieved after TUR-BT was submitted for NGS. The absence of FGFR genetic alteration did not support consideration of erdafitinib use. High mutation burden indicates the possibility of response to immune checkpoint blockade (5). Therefore, it is reasonable to deliver pembrolizumab as adjuvant treatment for the patients with UC who are cisplatin-ineligible after maximal TUR-BT. On the other hand, NGS also provides information clinical relevant genetic alterations. It could be inferred that genetic alterations with allele frequency around $50 \%$ are possible germline mutation. $\mathrm{CDH} 1$ gene, located at 16q22.1, encodes the cell-cell adhesion molecule protein called E-cadherin. It has been reported that CDH1 mutation was associated with hereditary diffuse type gastric cancer and lobular breast cancer. Some of the studies had found the genetic alteration was associated with bladder cancer. These alterations include CDH1 methylation, lossof-function mutation, and single nucleotide polymorphism (18-20). The patient was diagnosed a secondary diffuse type gastric cancer two years after the diagnosis of metastatic UC. Apart from tumor samples, CTC from liquid biopsy can also be used for genetic profiling. Furthermore, there were no sensitive and specific serum tumor markers in UC and CTC may be a serum biomarker to detect recurrent UC. In our case, CTC dynamics correlated with the disease 
course, and its elevation preceded image detection of bladder recurrence.

The reported case of metastatic UC was successfully treated with RAIT and therefore demonstrated the benefit of combining radiotherapy with immune checkpoint blockade. Although there were no prospective nor retrospective studies reported the toxicity profiles of RAIT. Our patients were well tolerated with RAIT with only grade 1 fatigue and pruritus noted. However, there were still some limitations in our study. Owing to lack of post-treatment sample after complete metabolic response, whether hypofractionated radiotherapy changed the tumor environment or not in our patient remains unclear.

In conclusion, this is a case of exceptional response and treating metastatic UC with RAIT in cisplatin-ineligible patients may warrant further investigation.

\section{Acknowledgments}

I would like to offer my special thanks to the colleagues of Taipei Cancer Center, molecular tumor board, Yun-Yen and Jackie Peng-Whang in Taipei Medical University for their valuable technical support on this project.

Funding: This research was supported by National Health Research Institute, Miaoli, Taiwan (grant No. NHRIEX107-10713EI-FY1), Taipei Medical University and TMU Hospital, Taipei, Taiwan (grant No. 105TMUCIT-02-1, 106 TMU-CIT-02-1, USTP-NTUTTMU-105-06, USTP-NTUT-TMU-106-01).

\section{Footnote}

Reporting Checklist: The authors have completed the CARE reporting checklist. Available at http://dx.doi.org/10.21037/ tro-20-30

Conflicts of Interest: All authors have completed the ICMJE uniform disclosure form (available at http://dx.doi. org/10.21037/tro-20-30). JFC serves as an unpaid editorial board member of Therapeutic Radiology and Oncology from Jun 2020 to May 2022. The other authors have no conflicts of interest to declare.

Ethical Statement: The authors are accountable for all aspects of the work in ensuring that questions related to the accuracy or integrity of any part of the work are appropriately investigated and resolved. All procedures performed in studies involving human participants were in accordance with the ethical standards of the institutional and/or national research committee(s) and with the Helsinki Declaration (as revised in 2013). Written informed consent was obtained from the patient.

Open Access Statement: This is an Open Access article distributed in accordance with the Creative Commons Attribution-NonCommercial-NoDerivs 4.0 International License (CC BY-NC-ND 4.0), which permits the noncommercial replication and distribution of the article with the strict proviso that no changes or edits are made and the original work is properly cited (including links to both the formal publication through the relevant DOI and the license). See: https://creativecommons.org/licenses/by-nc-nd/4.0/.

\section{References}

1. von der Maase H, Hansen SW, Roberts JT, et al. Gemcitabine and cisplatin versus methotrexate, vinblastine, doxorubicin, and cisplatin in advanced or metastatic bladder cancer: results of a large, randomized, multinational, multicenter, phase III study. J Clin Oncol 2000;18:3068-77.

2. Palma DA, Olson R, Harrow S, et al. Stereotactic ablative radiotherapy versus standard of care palliative treatment in patients with oligometastatic cancers (SABRCOMET): a randomised, phase 2, open-label trial. Lancet 2019;393:2051-8.

3. Loriot $\mathrm{Y}$, Necchi A, Park SH, et al. Erdafitinib in Locally Advanced or Metastatic Urothelial Carcinoma. N Engl J Med 2019;381:338-48.

4. Balar AV, Castellano D, O'Donnell PH, et al. First-line pembrolizumab in cisplatin-ineligible patients with locally advanced and unresectable or metastatic urothelial cancer (KEYNOTE-052): a multicentre, single-arm, phase 2 study. Lancet Oncol 2017;18:1483-92.

5. Balar AV, Galsky MD, Rosenberg JE, et al. Atezolizumab as first-line treatment in cisplatin-ineligible patients with locally advanced and metastatic urothelial carcinoma: a single-arm, multicentre, phase 2 trial. Lancet 2017;389:67-76.

6. Gettinger SN, Horn L, Gandhi L, et al. Overall Survival and Long-Term Safety of Nivolumab (Anti-Programmed Death 1 Antibody, BMS-936558, ONO-4538) in Patients With Previously Treated Advanced Non-Small-Cell Lung Cancer. J Clin Oncol 2015;33:2004-12.

7. Brahmer J, Reckamp KL, Baas P, et al. Nivolumab versus Docetaxel in Advanced Squamous-Cell Non-Small-Cell 
Lung Cancer. N Engl J Med 2015;373:123-35.

8. Galon J, Mlecnik B, Bindea G, et al. Towards the introduction of the 'Immunoscore' in the classification of malignant tumours. J Pathol 2014;232:199-209.

9. Galon J, Costes A, Sanchez-Cabo F, et al. Type, density, and location of immune cells within human colorectal tumors predict clinical outcome. Science 2006;313:1960-4.

10. Mandal R, Chan TA. Personalized Oncology Meets Immunology: The Path toward Precision Immunotherapy. Cancer Discov 2016;6:703-13.

11. Snyder A, Makarov V, Merghoub T, et al. Genetic basis for clinical response to CTLA-4 blockade in melanoma. $\mathrm{N}$ Engl J Med 2014;371:2189-99.

12. Obeid M, Tesniere A, Ghiringhelli F, et al. Calreticulin exposure dictates the immunogenicity of cancer cell death. Nat Med 2007;13:54-61.

13. Sundahl N, Vandekerkhove G, Decaestecker K, et al. Randomized Phase 1 Trial of Pembrolizumab with Sequential Versus Concomitant Stereotactic Body Radiotherapy in Metastatic Urothelial Carcinoma. Eur Urol 2019;75:707-11.

14. Xie G, Gu D, Zhang L, et al. A rapid and systemic complete response to stereotactic body radiation therapy and pembrolizumab in a patient with metastatic renal cell

doi: $10.21037 /$ tro-20-30

Cite this article as: Liu CS, Cheng CJ, Liu YR, Yeh SD, Jeng SC, Lu LS, Chiou JF. Complete response after radiationassisted immunotherapy for metastatic urothelial carcinoma: a case report and literature review. Ther Radiol Oncol 2020;4:23. carcinoma. Cancer Biol Ther 2017;18:547-51.

15. van Gysen K, Kneebone A, Eade T, et al. Advanced Renal Cell Cancer and Low-Dose Palliative Radiation Treatment: A Case of a Substantial and Sustained Treatment Response. Case Rep Oncol 2018;11:756-62.

16. LaPlant Q, Deselm C, Lockney NA, et al. Potential abscopal response to dual checkpoint blockade in RCC after reirradiation using dose-painting SBRT. Pract Radiat Oncol 2017;7:396-9.

17. Alexander GS, Palmer JD, Tuluc M, et al. Immune biomarkers of treatment failure for a patient on a phase I clinical trial of pembrolizumab plus radiotherapy. J Hematol Oncol 2016;9:96.

18. Maruyama R, Toyooka S, Toyooka KO, et al. Aberrant promoter methylation profile of bladder cancer and its relationship to clinicopathological features. Cancer Res 2001;61:8659-63.

19. Al-Ahmadie HA, Iyer G, Lee BH, et al. Frequent somatic CDH1 loss-of-function mutations in plasmacytoid variant bladder cancer. Nat Genet 2016;48:356-8.

20. Kiemeney LA, van Houwelingen KP, Bogaerts M, et al. Polymorphisms in the E-cadherin (CDH1) gene promoter and the risk of bladder cancer. Eur J Cancer 2006;42:3219-27. 